\title{
EFFECT OF ACTIVE AND PASSIVE MODIFIED ATMOSPHERE PACKAGING ON QUALITY ATTRIBUTES OF BROCCOLI FLORETS \\ Mohamed, M. E. ${ }^{1}$; M. E. Ragab ${ }^{2}$; S. M. El-miniawy ${ }^{2}$ and Rawia E. I. El-Bassiouny ${ }^{1}$ \\ 1-Postharvest and Handling of Vegetable Crops Dept., Hort. Res. Inst., Agric. Res. Center, Dokki, Giza, Egypt.
}

2- Dept. of Horticulture, Fac. of Agric., Ain Shams Univ., Shoubra ElKheima, Cairo, Egypt.

\begin{abstract}
The experiment was carried out during the two seasons of 2008 and 2009 on broccoli $F_{1}$ Hybrid Sakura to study the effect of two packaging materials and modified atmosphere packaging (MAP) on quality attributes of broccoli florets stored at $0^{\circ} \mathrm{C}$ and $95 \% \mathrm{RH}$ for 16 days.

Broccoli florets packed in polypropylene film showed the highest intensities of freshness, greenness, and compactness and had less off-odor as compared with those stored in polyethylene bags. There were no differences in weight loss between broccoli florets stored in the different packaging materials.

Florets packed in active MAP retained their weight during storage as compared to those in passive MAP. The optimum gas composition of MAP tests for maintaining quality of broccoli florets during storage was $5 \% \mathrm{O}_{2}+10 \% \mathrm{Co}_{2}$.

Off-odor was not observed in broccoli florets packed in polypropylene film and exposed to $5 \% \mathrm{O}_{2}+5 \% \mathrm{Co}_{2}$, while at $5 \% \mathrm{O}_{2}+10 \% \mathrm{Co}_{2}$ gave a slight off-odor at the end of storage with non significant differences between them.

Packing broccoli florets packed in polypropylene film and exposing to active MAP $\left(5 \% \mathrm{O}_{2}+10 \% \mathrm{Co}_{2}\right)$ was the most effective treatment for reducing weight loss and color change and maintaining high content of chlorophyll and gave florets with good appearance for 16 days at $0^{\circ} \mathrm{C}$ and $95 \% \mathrm{RH}$.
\end{abstract}

\section{INTRODUCTION}

Broccoli has a relatively high respiration and transpiration rates and a short shelf life it is also, extremely sensitive to ethylene and loss water rapidly (Brennan and Shewfelt, 1989). The shelf life of florets was 1-3 days at $20^{\circ} \mathrm{C}$ (Wang and Hruschka, 1977). Refrigeration is the primary means that maintaining broccoli in good condition for 3 weeks of storage when held at 0 $2{ }^{\circ} \mathrm{C}$ (Ryall and Lipton, 1979). In addition to refrigeration, modified atmosphere packaging (MAP) is commonly used to maintain the quality and improve the shelf life of broccoli florets (Charles et al., 1991). The MAP of broccoli at elevated $\mathrm{CO}_{2}$ and reduced $\mathrm{O}_{2}$ levels has been shown to retard deterioration, i.e. yellowing and softening, and preserve the market quality of broccoli during storage (Barth et al., 1993, Elkashif et al., 1993 and Jacobsson et al., 2004) The low $\mathrm{O}_{2}$ and high $\mathrm{Co}_{2}$ levels that are modified through the product's respiration and the permeability of the film slow down

This research was supported by Development of Postharvest Treatments Project. 
respiration and have an effect on inhibiting microbial growth in MAP ( Hu et al., 2007). Broccoli can be stored at 1 to $2 \% \mathrm{O}_{2}$ and 5 to $10 \% \mathrm{Co}_{2}$ atmospheres at low temperatures (Jacobsson et al., 2003). However, a low oxygen level $(0.5-2 \%)$ or carbon dioxide concentration in excess of $10 \%$, combined with temperature fluctuations, may results in the production of offodors, thus reducing the shelf life of the broccoli (Ballantyne et al., 1988 and Makhlouf et al., 1989).

The main benefit, however, of using MAP is to inhibit decay and generation of $\mathrm{C}_{2} \mathrm{H}_{4}$ because it can influence the enzymes activity, thus the permeability of cell membranes does not increase quickly (Ballantyne et al., 1988). Furthermore MAP can prevent microbial growth, discoloration and cell destruction and can reduce the risk of infection (Barth et al., 1993)

The objective of this present work was to evaluate the potential of active and passive modified atmosphere packaging in preserving the quality and extend the shelf life of broccoli florets during storage at $0^{\circ} \mathrm{C}$.

\section{MATERIALS AND METHODS}

Seeds of broccoli (Brassica Oleracea var. italica) $F_{1}$ Hybrid Sakura (seeds from Tokita Co, Japan) were sown at the nursery in transplanting trays on September $5^{\text {th }}$ and $8^{\text {th }} 2009$ and 2010 respectively. The transplants were set up in the field on October $4^{\text {th }}$ and $8^{\text {th }}$ in the first and the second seasons, respectively, at the Agricultural Experiment and Research Station, Faculty of Agriculture, Cairo University. The soil was loamy clay. The agriculture practices took place wherever necessary according to the recommendations of Ministry of Agriculture.

Broccoli heads were harvested at the proper stage of marketing on $2^{\text {nd }}$ and $5^{\text {th }}$ of December 2009 and 2010, respectively, and then transported immediately to the laboratory of postharvest center, Horticulture Research Institute, Giza. Heads were separated to florets which were rinsed in chlorinated water (1000 ppm as sodium hypo chloride) for $10 \mathrm{~min}$.

Two commercially available polymeric films/ bags were used as packaging materials. The investigated polymer materials were polypropylene and polyethylene $(15 \times 20 \mathrm{~cm})$ of $40 \mu \mathrm{m}$ thickness. Broccoli florets were packaged, each package contained 200 grams as one replicate. The packages were all heat sealed and treated as follows:

1- Passive MAP (A)

2- Flushed with a gas mixture (active MAP) $5 \% \mathrm{O}_{2}+5 \% \mathrm{CO}_{2}$ (B)

3- Flushed with a gas mixture (active MAP) $5 \% \mathrm{O}_{2}+10 \% \mathrm{CO}_{2}$ (C)

Twenty four replicate were prepared for each treatment. The samples were arranged in a complete randomized design and stored at $0^{\circ} \mathrm{C}$ and $95 \%$ R.H. for 16 days. The treatments were examined immediately after harvest and every 4 days intervals for and the following parameters were determined:

1) Weight loss percentage.

2) General appearance $(G A)$ :

GA was determined according to the following score

system: $9=$ excellent, $7=$ good, $5=$ fair, $3=$ poor

and $1=$ unusable. The scale depends on morphological 
defects such as shriveling fresh appearance, color change of florets and decay. Florets rating (5) or below considered unmarketable.

3) Off odor:

Off odor was evaluated on a scale of 1 to 5 where $1=$ none, $2=$ slight, $3=$ moderate, $4=$ sever and $5=$ extremely sever.

4) External surface color:

Color was evaluated by a color difference meter (Minolta CR400) to measure the $L$ and hue angle value.

5) Total chlorophyll:

Total chlorophyll was determined according to A.O.A.C. (1990).

6) Gas composition inside the packages: the concentration of $\mathrm{O}_{2}$ and $\mathrm{Co}_{2}$ inside the packages were monitored using Dual Trak model $902 \mathrm{D}$ gas analyzer and expressed by percentage. By inserting the test probe through a rubber seal attached to the outside of the packaging.

All obtained data were statically analyzed according to the method described by Snedecor and Cochran (980).

\section{RESULTS AND DISCUSSION}

Weight loss:

Data in Table (1) show that weight loss percentage of broccoli florets increased considerably and consistently with the prolongation of storage period. The loss in weight may be attributed to respiration and other senescence related metabolic processes during storage (Charles et al., 1991).

As to packaging material, no significant differences were found in weight loss percentage between polypropylene and polyethylene packages. However, there were significant differences among MAP treatments. Broccoli florets held in active MAP retained their weight during storage as compared with passive MAP. Moreover, active MAP of $5 \% \mathrm{O}_{2}+10 \% \mathrm{Co}_{2}$ resulted in prominent reduction in weight loss percentage. Joseph and Mishael (1992) found that reducing $\mathrm{O}_{2}$ concentration to $2 \%$ and increasing $\mathrm{Co}_{2}$ to $5 \%$ resulted in more than 10 fold decline in the rate respiration and reduce sensitivity of ethylene of broccoli which diminished the weight loss in the florets during storage (Jacobsson and Nielsen, 2003).

As for the interaction between packaging materials, MAP treatments and storage period, data in Table (1) show that broccoli florets packed in polypropylene or polyethylene bags with active MAP at $5 \% \mathrm{O}_{2}+10 \% \mathrm{Co}_{2}$ had the lowest weight loss percent during all storage period. 
Table(1): Effect of active and passive modified atmosphere packages (MAP) on weight loss percentage of broccoli florets during storage in 2009 and 2010 seasons.

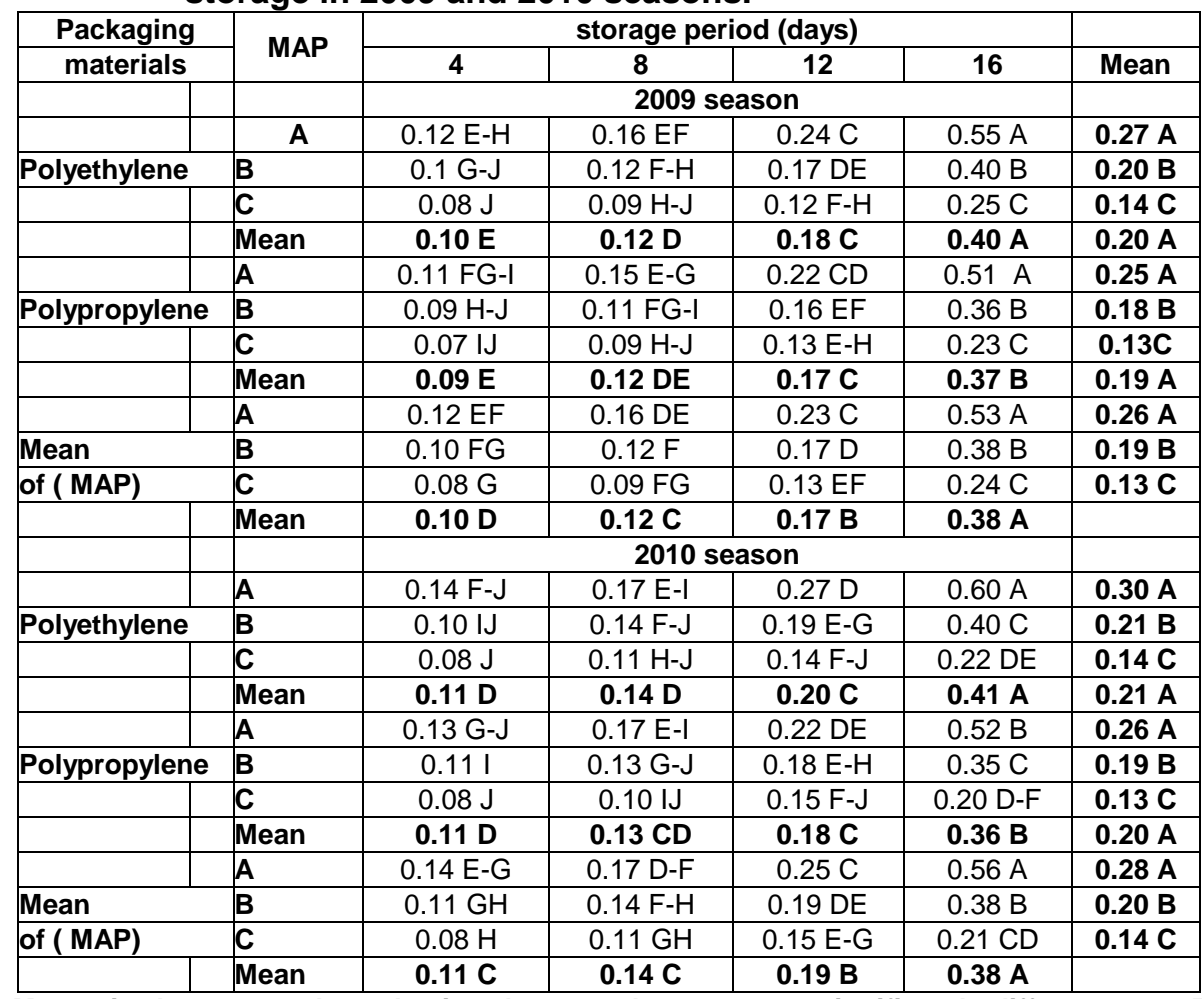

Means in the same column having the same letter are not significantly different at 0.05 level by Duncan's multiple rang test.

A=Passive MAP

B=Active MAP(5\%02+5\%Co2)

C=Active MAP(5\%02+10\%Co2)

\section{General appearance:}

Data in Table (2) show that general appearance (GA) of broccoli florets decreased with the prolongation of storage period. Similar results were reported by Jacobsson et al. (2004). The decrease of GA during storage period might be due to shrivelling, color change and less compact (Forney and Rij, 1991).

Significant differences in appearance were found between the two packaging materials on the broccoli. Broccoli florets stored in polypropylene bags were perceived the higher intensities of freshness, greenness and compactness as compared with those stored in polyethylene bags.

Concerning MAP effect the general appearance of broccoli florets exposed to active MAP was better than passive MAP. Active MAP for $5 \% \mathrm{O}_{2}+$ $10 \% \mathrm{Co}_{2}$ showed the best appearance because it does not exhibit any changes in their appearance till the $12^{\text {th }}$ days at $0^{\circ} \mathrm{C}$ and gave product with good appearance at the end of storage (16 days). While using $5 \% \mathrm{O}_{2}+5 \%$ 
$\mathrm{Co}_{2}$ rated good appearance after 12 days from storage. On the other hand, passive MAP rated excellent appearance after 4 days and dropped to poor appearance at the end of storage.

The interaction among packaging materials, MAP treatments and storage period revealed that broccoli florets packed in polypropylene or polyethylene film and exposed to active MAP at $5 \% \mathrm{O}_{2}+10 \% \mathrm{Co}_{2}$ performed good appearance after 16 days of storage at $0^{\circ} \mathrm{C}$.

Table(2): Effect of active and passive modified atmosphere packages (MAP) on general appearance(score) of broccoli florets during storage in 2009 and 2010 seasons.

\begin{tabular}{|c|c|c|c|c|c|c|c|}
\hline \multirow{3}{*}{$\begin{array}{c}\text { Packaging } \\
\text { materials }\end{array}$} & \multirow{2}{*}{ MAP } & \multicolumn{4}{|c|}{ storage period (days) } & \multicolumn{2}{|c|}{ Mean } \\
\hline & & 0 & 4 & 8 & 12 & 16 & \\
\hline & & \multicolumn{4}{|c|}{2009 season } & & \\
\hline & A & $9.00 \mathrm{~A}$ & $9.00 \mathrm{~A}$ & $6.33 \mathrm{DE}$ & $5.67 \mathrm{E}$ & $4.33 \mathrm{~F}$ & 6.87E \\
\hline \multirow[t]{4}{*}{ Polyethylene } & B & $9.00 \mathrm{~A}$ & $9.00 \mathrm{~A}$ & $7.67 \mathrm{CD}$ & $7.00 \mathrm{DE}$ & $5.33 \mathrm{~F}$ & $7.6 \mathrm{CD}$ \\
\hline & C & $9.00 \mathrm{~A}$ & $9.00 \mathrm{~A}$ & $8.33 \mathrm{BC}$ & $7.67 \mathrm{CD}$ & $7.00 \mathrm{EF}$ & $8.2 \mathrm{AB}$ \\
\hline & Mean & $9.00 \mathrm{~A}$ & $9.00 \mathrm{~A}$ & $7.44 \mathrm{BC}$ & 6.78 CD & $5.55 \mathrm{E}$ & $7.56 \mathrm{~B}$ \\
\hline & A & $9.00 \mathrm{~A}$ & $9.00 \mathrm{~A}$ & $7.00 \mathrm{AB}$ & $6.33 \mathrm{BC}$ & $4.33 \mathrm{DE}$ & 7.13 DE \\
\hline \multirow[t]{4}{*}{ Polypropylene } & B & $9.00 \mathrm{~A}$ & $9.00 \mathrm{~A}$ & $8.33 \mathrm{AB}$ & $7.67 \mathrm{BC}$ & $6.33 \mathrm{CD}$ & $8.07 \mathrm{BC}$ \\
\hline & C & $9.00 \mathrm{~A}$ & $9.00 \mathrm{~A}$ & $9.00 \mathrm{~A}$ & 8.67 AB & $7.67 \mathrm{BC}$ & $8.67 \mathrm{~A}$ \\
\hline & Mean & $9.00 \mathrm{~A}$ & $9.00 \mathrm{~A}$ & $8.11 \mathrm{~B}$ & $7.56 \mathrm{~B}$ & 6.11 DE & $7.96 \mathrm{~A}$ \\
\hline & A & $9.00 \mathrm{~A}$ & $9.00 \mathrm{~A}$ & 6.67 DE & $6.00 \mathrm{E}$ & $4.33 \mathrm{~F}$ & $7.00 \mathrm{C}$ \\
\hline Mean & B & $9.00 \mathrm{~A}$ & $9.00 \mathrm{~A}$ & $8.00 \mathrm{BC}$ & $7.34 \mathrm{CD}$ & $5.83 \mathrm{E}$ & $7.83 \mathrm{~B}$ \\
\hline \multirow{4}{*}{ of ( MAP) } & C & $9.00 \mathrm{~A}$ & $9.00 \mathrm{~A}$ & $8.67 \mathrm{AB}$ & $8.17 \mathrm{~A}-\mathrm{C}$ & $7.34 \mathrm{CD}$ & $8.43 \mathrm{~A}$ \\
\hline & Mean & $9.00 \mathrm{~A}$ & $9.00 \mathrm{~A}$ & $7.78 \mathrm{~B}$ & $7.17 \mathrm{C}$ & $5.83 \mathrm{D}$ & \\
\hline & & \multicolumn{4}{|c|}{2010 season } & & \\
\hline & A & $9.00 \mathrm{~A}$ & $9.00 \mathrm{~A}$ & 5.67 EF & 5.00 FG & $4.33 \mathrm{G}$ & $6.60 \mathrm{D}$ \\
\hline \multirow{4}{*}{\begin{tabular}{|l} 
Polyethylene \\
\end{tabular}} & B & $9.00 \mathrm{~A}$ & $9.00 \mathrm{~A}$ & $7.33 \mathrm{BCD}$ & $7.00 \mathrm{CD}$ & $6.33 \mathrm{DE}$ & $7.73 \mathrm{~B}$ \\
\hline & C & $9.00 \mathrm{~A}$ & $9.00 \mathrm{~A}$ & $8.33 \mathrm{AB}$ & $7.67 \mathrm{BC}$ & $7.00 \mathrm{CD}$ & $8.20 \mathrm{AB}$ \\
\hline & Mean & $9.00 \mathrm{~A}$ & $9.00 \mathrm{~A}$ & 7.11 CD & $6.56 \mathrm{DE}$ & $5.89 \mathrm{~F}$ & $7.51 \mathrm{~B}$ \\
\hline & A & $9.00 \mathrm{~A}$ & $9.00 \mathrm{~A}$ & $7.00 \mathrm{CD}$ & $6.33 \mathrm{DE}$ & $4.33 \mathrm{G}$ & $7.13 \mathrm{C}$ \\
\hline \multirow[t]{4}{*}{ Polypropylene } & B & $9.00 \mathrm{~A}$ & $9.00 \mathrm{~A}$ & $7.67 \mathrm{BC}$ & $7.00 \mathrm{CD}$ & $6.33 \mathrm{DE}$ & $7.80 \mathrm{~B}$ \\
\hline & C & $9.00 \mathrm{~A}$ & $9.00 \mathrm{~A}$ & $9.00 \mathrm{~A}$ & $8.33 \mathrm{AB}$ & $7.33 \mathrm{~B}-\mathrm{D}$ & $8.53 \mathrm{~A}$ \\
\hline & Mean & $9.00 \mathrm{~A}$ & $9.00 \mathrm{~A}$ & $7.89 \mathrm{~B}$ & $7.22 \mathrm{C}$ & $6.00 \mathrm{G}$ & $7.82 \mathrm{~A}$ \\
\hline & A & $9.00 \mathrm{~A}$ & $9.00 \mathrm{~A}$ & $6.34 \mathrm{EF}$ & $5.67 \mathrm{~F}$ & $4.33 \mathrm{G}$ & $6.87 \mathrm{C}$ \\
\hline Mean & B & $9.00 \mathrm{~A}$ & $9.00 \mathrm{~A}$ & $7.50 \mathrm{CD}$ & 7.00 DE & $6.33 \mathrm{EF}$ & $7.77 \mathrm{~B}$ \\
\hline \multirow{2}{*}{ of ( MAP) } & C & $9.00 \mathrm{~A}$ & $9.00 \mathrm{~A}$ & $8.67 \mathrm{AB}$ & $8.00 \mathrm{BC}$ & $7.17 \mathrm{D}$ & $8.37 \mathrm{~A}$ \\
\hline & Mean & 9.0 A & $9.0 \mathrm{~A}$ & $7.50 \mathrm{~B}$ & $6.89 \mathrm{C}$ & 5.94 D & \\
\hline
\end{tabular}

Means in the same column having the same letter are not significantly different at 0.05 level by Duncan's multiple rang test.

A=Passive MAP

B=Active MAP(5\%02+5\%Co2)

C=Active MAP(5\%02+10\%Co2)

Off-odor:

Data in Table (3) show that off-odor inside the different packaging materials started to be observed after 8 days of storage, and then increased till the end of storage period.

These strong off-odors have mainly been associated with sulphur volatile compound, for example, methanethiol, hydrogen sulphide, dimethyl disulphide 
and dimethyl trisulphide (Hansen et al., 1992) However, off-odor was not observed in broccoli florets packed in polypropylene film and exposed to $5 \%$ $\mathrm{O}_{2}+5 \% \mathrm{Co}_{2}$, while those exposed to $5 \% \mathrm{O}_{2}+10 \% \mathrm{Co}_{2}$ or passive MAP gave a slight off-odor at the end of storage. However, no significant differences were detected between both active MAP treatments Broccoli florets packed in polyethylene film with MAP gave the highest score of off-odor especially at $5 \%$ $\mathrm{O}_{2}+10 \% \mathrm{Co}_{2}$ after 16 days of storage. These results agree with those obtained by Hansen et al.(1993) who found that the off-odor developed when the storage atmosphere contained a high level of $\mathrm{Co}_{2}$ in combination with very low $\mathrm{O}_{2}$ concentration.

Table(3): Effect of active and passive modified atmosphere packages (MAP) on off-odor (score) of broccoli florets during storage in 2009 and 2010 seasons.

\begin{tabular}{|c|c|c|c|c|c|c|c|}
\hline \multirow{3}{*}{$\begin{array}{c}\text { Packaging } \\
\text { materials }\end{array}$} & \multirow{2}{*}{ MAP } & \multicolumn{5}{|c|}{ storage period (days) } & \multirow{2}{*}{ Mean } \\
\hline & & 0 & 4 & 8 & 12 & 16 & \\
\hline & & \multicolumn{5}{|c|}{2009 season } & \\
\hline & A & $1.00 \mathrm{D}$ & $1.00 \mathrm{D}$ & $1.33 \mathrm{CD}$ & $1.67 \mathrm{CD}$ & $2.00 \mathrm{BC}$ & $1.40 \mathrm{~B}$ \\
\hline \multirow[t]{4}{*}{ Polyethylene } & B & $1.00 \mathrm{D}$ & $1.00 \mathrm{D}$ & $1.00 \mathrm{D}$ & $2.00 \mathrm{BC}$ & $3.67 \mathrm{~A}$ & $1.73 \mathrm{~A}$ \\
\hline & C & $1.00 \mathrm{D}$ & $1.00 \mathrm{D}$ & $1.67 \mathrm{CD}$ & $2.67 \mathrm{~B}$ & $3.67 \mathrm{~A}$ & $2.00 \mathrm{~A}$ \\
\hline & Mean & $1.00 \mathrm{D}$ & $1.00 \mathrm{D}$ & $1.33 \mathrm{CD}$ & $2.11 \mathrm{~B}$ & $3.11 \mathrm{~A}$ & $1.71 \mathrm{~A}$ \\
\hline & A & $1.00 \mathrm{D}$ & $1.00 \mathrm{D}$ & $1.00 \mathrm{D}$ & $1.33 \mathrm{CD}$ & $1.67 \mathrm{CD}$ & $1.20 \mathrm{BC}$ \\
\hline \multirow[t]{4}{*}{ Polypropylene } & B & $1.00 \mathrm{D}$ & $1.00 \mathrm{D}$ & $1.00 \mathrm{D}$ & $1.00 \mathrm{D}$ & $1.00 \mathrm{D}$ & $1.00 \mathrm{C}$ \\
\hline & C & $1.00 \mathrm{D}$ & $1.00 \mathrm{D}$ & $1.00 \mathrm{D}$ & $1.00 \mathrm{D}$ & $1.67 \mathrm{CD}$ & $1.13 \mathrm{BC}$ \\
\hline & Mean & $1.00 \mathrm{D}$ & $1.00 \mathrm{D}$ & $1.00 \mathrm{D}$ & $1.11 \mathrm{CD}$ & $1.45 \mathrm{C}$ & $1.11 \mathrm{~B}$ \\
\hline & A & $1.00 \mathrm{D}$ & $1.00 \mathrm{D}$ & 1.17 CD & $1.50 \mathrm{BC}$ & $1.84 \mathrm{~B}$ & $1.30 \mathrm{~B}$ \\
\hline Mean & B & $1.00 \mathrm{D}$ & $1.00 \mathrm{D}$ & $1.00 \mathrm{D}$ & $1.50 \mathrm{DC}$ & $2.34 \mathrm{~A}$ & $1.37 \mathrm{AB}$ \\
\hline \multirow{4}{*}{ of ( MAP) } & C & $1.00 \mathrm{D}$ & $1.00 \mathrm{D}$ & $1.34 \mathrm{CD}$ & $1.84 \mathrm{~B}$ & $2.67 \mathrm{~A}$ & $1.57 \mathrm{~A}$ \\
\hline & Mean & $1.00 \mathrm{D}$ & $1.00 \mathrm{D}$ & $1.17 \mathrm{CD}$ & $1.61 \mathrm{~B}$ & $2.28 \mathrm{~A}$ & \\
\hline & \multicolumn{6}{|c|}{2010 season } & \\
\hline & A & $1.00 \mathrm{~F}$ & $1.00 \mathrm{~F}$ & 1.67 D-F & $2.00 \mathrm{C}-\mathrm{E}$ & $2.33 \mathrm{CD}$ & $1.60 \mathrm{BC}$ \\
\hline \multirow[t]{4}{*}{ Polyethylene } & B & $1.00 \mathrm{~F}$ & $1.00 \mathrm{~F}$ & $1.00 \mathrm{~F}$ & $2.67 \mathrm{BC}$ & $3.33 \mathrm{AB}$ & $1.80 \mathrm{AB}$ \\
\hline & C & $1.00 \mathrm{~F}$ & $1.00 \mathrm{~F}$ & \begin{tabular}{|l}
$1.33 \mathrm{EF}$ \\
\end{tabular} & $2.67 \mathrm{BC}$ & $3.67 \mathrm{~A}$ & $1.93 \mathrm{~A}$ \\
\hline & Mean & $1.00 \mathrm{D}$ & $1.00 \mathrm{D}$ & \begin{tabular}{|l|}
$1.33 \mathrm{CD}$ \\
\end{tabular} & $2.45 \mathrm{~B}$ & $3.11 \mathrm{~A}$ & $1.78 \mathrm{~A}$ \\
\hline & A & $1.00 \mathrm{~F}$ & $1.00 \mathrm{~F}$ & $1.00 \mathrm{~F}$ & $1.67 \mathrm{D}-\mathrm{F}$ & $2.00 \mathrm{C}-\mathrm{E}$ & $1.33 \mathrm{CD}$ \\
\hline \multirow[t]{4}{*}{ Polypropylene } & B & $1.00 \mathrm{~F}$ & $1.00 \mathrm{~F}$ & $1.00 \mathrm{~F}$ & $1.00 \mathrm{~F}$ & $1.00 \mathrm{~F}$ & $1.00 \mathrm{E}$ \\
\hline & C & $1.00 \mathrm{~F}$ & $1.00 \mathrm{~F}$ & $1.00 \mathrm{~F}$ & $1.33 \mathrm{EF}$ & $1.67 \mathrm{D}-\mathrm{F}$ & $1.20 \mathrm{DE}$ \\
\hline & Mean & $1.00 \mathrm{D}$ & $1.00 \mathrm{D}$ & $1.00 \mathrm{D}$ & $1.33 \mathrm{CD}$ & $1.56 \mathrm{C}$ & $1.18 \mathrm{~B}$ \\
\hline & A & $1.00 \mathrm{C}$ & $1.00 \mathrm{C}$ & $1.34 \mathrm{C}$ & $1.84 \mathrm{~B}$ & $2.17 \mathrm{~B}$ & $1.47 \mathrm{~A}$ \\
\hline Mean & B & $1.00 \mathrm{C}$ & $1.00 \mathrm{C}$ & $1.00 \mathrm{C}$ & $1.84 \mathrm{~B}$ & $2.17 \mathrm{~B}$ & $1.40 \mathrm{~A}$ \\
\hline \multirow[t]{2}{*}{ of ( MAP) } & C & $1.00 \mathrm{C}$ & $1.00 \mathrm{C}$ & $1.17 \mathrm{C}$ & $2.00 \mathrm{~B}$ & $2.67 \mathrm{~A}$ & $1.57 \mathrm{~A}$ \\
\hline & Mean & $1.0 \mathrm{C}$ & $1.00 \mathrm{C}$ & $1.17 \mathrm{C}$ & $1.89 \mathrm{~B}$ & $2.33 \mathrm{~A}$ & \\
\hline
\end{tabular}

Means in the same column having the same letter are not significantly different at 0.05 level by Duncan's multiple rang test.

A=Passive MAP

B=Active MAP $(5 \% 02+5 \%$ Co2 $)$

C=Active MAP(5\%02+10\%Co2)

\section{Color:}

The color of the homogenized samples was measured recording $L$ value and hue angle. The $L$ value is measure of the lightness of the florets, while the hue angle represents acoordinat in a standardized color space. 
Changes in lightness $(L)$ and hue angle $\left(h^{\circ}\right)$ values were observed during storage compared to initial values (Table 4). Lightness of broccoli florets was affected by storage time. An increment in $L$ value was detected during by prolonging the storage period. Broccoli backed in the two packaging material, in addition to active MAP at $5 \% \mathrm{O}_{2}+10 \% \mathrm{Co}_{2}$ reduced this increases in $\mathrm{L}$ value and remained very close to the initial value during storage, resulted in darker color (lower $\mathrm{L}$ value). These results are in agreement with those obtained by Charles et al. (1991).

Table(4): Effect of active and passive modified atmosphere packages (MAP) on color (L.Value) of broccoli florets during storage in 2009 and 2010 seasons.

\begin{tabular}{|c|c|c|c|c|c|c|c|}
\hline \multirow{2}{*}{$\begin{array}{l}\text { Packaging } \\
\text { materials }\end{array}$} & \multirow{2}{*}{ MAP } & \multicolumn{5}{|c|}{ / storage period (days) } & \multirow{2}{*}{ Mean } \\
\hline & & $\begin{array}{lll}0 \\
\end{array}$ & 4 & 8 & 12 & 16 & \\
\hline & & \multicolumn{5}{|c|}{2009 season } & \\
\hline & A & $41.00 \mathrm{~N}$ & $41.58 \mathrm{H}-\mathrm{M}$ & 42.47 DEF & $43.67 \mathrm{~B}$ & $45.73 \mathrm{~A}$ & $42.89 \mathrm{~A}$ \\
\hline \multirow[t]{4}{*}{ Polyethylene } & B & $41.00 \mathrm{~N}$ & $41.31 \mathrm{MN}$ & $41.93 \mathrm{~F}-\mathrm{K}$ & $42.15 \mathrm{E}-\mathrm{H}$ & $42.85 \mathrm{D}$ & $41.85 \mathrm{~B}$ \\
\hline & C & $41.00 \mathrm{~N}$ & $41.18 \mathrm{MN}$ & $41.60 \mathrm{H}-\mathrm{M}$ & $41.88 \mathrm{G}-\mathrm{L}$ & \multicolumn{2}{|l|}{$42.23 \mathrm{E}-\mathrm{G}$} \\
\hline & Mean & $41.00 \mathrm{G}$ & $41.36 \mathrm{~F}$ & 42.00 DE & $42.57 \mathrm{C}$ & $43.60 \mathrm{~A}$ & $42.11 \mathrm{~A}$ \\
\hline & A & $41.00 \mathrm{~N}$ & $41.44 \mathrm{~J}-\mathrm{N}$ & $42.61 \mathrm{DE}$ & $43.19 \mathrm{BC}$ & $45.26 \mathrm{~A}$ & $42.70 \mathrm{~A}$ \\
\hline \multirow[t]{4}{*}{ Polypropylene } & B & $41.00 \mathrm{~N}$ & $41.21 \mathrm{MN}$ & $41.53 \mathrm{I}-\mathrm{N}$ & $42.03 \mathrm{~F}-\mathrm{I}$ & \multicolumn{2}{|c|}{ 42.48 DEF 41.65 BC } \\
\hline & C & $41.00 \mathrm{~N}$ & $41.07 \mathrm{MN}$ & $41.23 \mathrm{MN}$ & $41.40 \mathrm{~K}-\mathrm{N}$ & $42.00 \mathrm{~F}-\mathrm{J}$ & 41.34 D \\
\hline & Mean & $41.00 \mathrm{G}$ & 41.24 FG & 41.79 E & 42.21 D & 43.25 B & $41.90 \mathrm{~B}$ \\
\hline & A & $41.00 \mathrm{H}$ & $41.51 \mathrm{E}-\mathrm{G}$ & $42.54 \mathrm{C}$ & $43.43 \mathrm{~B}$ & $54.50 \mathrm{~A}$ & 42.80 A \\
\hline Mean & B & $41.00 \mathrm{H}$ & $41.26 \mathrm{~F}-\mathrm{H}$ & $41.73 \mathrm{BE}$ & $42.09 \mathrm{D}$ & $42.67 \mathrm{C}$ & 41.75 B \\
\hline \multirow[t]{4}{*}{ of ( MAP) } & C & $41.00 \mathrm{H}$ & $41.13 \mathrm{GH}$ & 41.42 EFG & 41.64 EF & 42.12 D & $41.46 \mathrm{C}$ \\
\hline & Mean & $41.00 \mathrm{E}$ & 41.30 D & $41.90 \mathrm{C}$ & 42.39 B & 43.43 A & \\
\hline & \multicolumn{7}{|c|}{2010 season } \\
\hline & A & $41.32 \mathrm{~N}$ & 42.61 H-M & 43.52 DE & $44.72 \mathrm{~B}$ & $46.87 \mathrm{~A}$ & 43.81 A \\
\hline \multirow[t]{4}{*}{ Polyethylene } & B & $41.32 \mathrm{~N}$ & $42.33 \mathrm{~J}-\mathrm{M}$ & $42.98 \mathrm{E}-\mathrm{I}$ & $43.20 \mathrm{E}-\mathrm{G}$ & $43.91 \mathrm{CD}$ & 42.75 B \\
\hline & C & $41.32 \mathrm{~N}$ & $42.20 \mathrm{LM}$ & 42.64 G-M & 42.91 F-J & $43.25 \mathrm{E}-\mathrm{G}$ & $42.46 \mathrm{C}$ \\
\hline & Mean & $41.32 \mathrm{G}$ & $42.38 \mathrm{~F}$ & 43.05 DE & 43.61 C & $44.68 \mathrm{~A}$ & 43.01 A \\
\hline & A & $41.32 \mathrm{~N}$ & $42.34 \mathrm{~J}-\mathrm{M}$ & $43.11 \mathrm{E}-\mathrm{H}$ & $44.12 \mathrm{C}$ & $47.00 \mathrm{~A}$ & $43.58 \mathrm{~A}$ \\
\hline \multirow[t]{4}{*}{ Polypropylene } & B & $41.32 \mathrm{~N}$ & 42.23 K-M & 42.74 F-L & $43.07 \mathrm{E}-\mathrm{H}$ & \multicolumn{2}{|c|}{$43.20 \mathrm{E}-\mathrm{G} \quad 42.51 \mathrm{BC}$} \\
\hline & C & $41.32 \mathrm{~N}$ & $42.10 \mathrm{M}$ & $42.44 \mathrm{I}-\mathrm{M}$ & $42.30 \mathrm{KLM}$ & 42.81 F-K & 42.19 D \\
\hline & Mean & $41.32 \mathrm{G}$ & $42.22 \mathrm{~F}$ & 42.76 E & 43.16 D & 44.34 B & 42.76 B \\
\hline & A & 41.32 I & 42.48 FGH & $43.32 \mathrm{CD}$ & $44.42 \mathrm{~B}$ & $46.94 \mathrm{~A}$ & 43.69 A \\
\hline Mean & B & 41.32 I & $42.28 \mathrm{GH}$ & $42.86 \mathrm{EF}$ & 43.14 DE & $43.56 \mathrm{C}$ & 42.63 B \\
\hline \multirow[t]{2}{*}{ of ( MAP) } & C & 41.32 I & $42.15 \mathrm{H}$ & $42.547 \mathrm{~F}-\mathrm{H}$ & 42.61 FG & 43.03 DE & $42.33 \mathrm{C}$ \\
\hline & Mean & $41.32 \mathrm{E}$ & 42.30 D & $42.91 \mathrm{C}$ & 43.39 B & 44.15 A & \\
\hline
\end{tabular}

Means in the same column having the same letter are not significantly different at 0.05 level by Duncan's multiple rang test.

\section{A=Passive MAP}

B=Active MAP $(5 \% 02+5 \%$ Co2 $)$

C=Active MAP(5\%02+10\%Co2)

Hue angle $\left(h^{\circ}\right)$ gave the best indication of greenness. Data in Table (5) indicate that there was a decrease in hue angle value during storage, however, broccoli florets packed in polypropylene film were more green (higher hue angle value) as compared with polyethylene film. On the other hand, broccoli stored at passive MAP gave the lower value of hue angle as an 
important degreening or intense yellowing compared to active MAP. In addition active MAP at $5 \% \mathrm{O}_{2}+10 \% \mathrm{Co}_{2}$ did no show the same behavior and maintained high hue angle values during storage. The treatments that showed most retention of green color (high hue angle) were performed with broccoli florets packed in polypropylene film plus active MAP at $5 \% \mathrm{O}_{2}+10 \% \mathrm{Co}_{2}$ These results agree with those obtained by Jacobsson and Nielsen (2003) who found that MAP maintain the quality and extend the shelf life of broccoli florets by delaying softening and color changes.

Table(5): Effect of active and passive modified atmosphere packages (MAP) on color (hue angle $\mathrm{h}^{\circ}$ ) of broccoli florets during storage in 2009 and 2010 seasons.

\begin{tabular}{|c|c|c|c|c|c|c|c|}
\hline \multirow{2}{*}{$\begin{array}{l}\text { Packaging } \\
\text { materials }\end{array}$} & \multirow[b]{2}{*}{ MAP } & \multicolumn{5}{|c|}{ storage period (days) } & \multirow[b]{2}{*}{ Mean } \\
\hline & & 0 & 4 & 8 & 12 & 16 & \\
\hline & & \multicolumn{5}{|c|}{2009 season } & \\
\hline & A & $125.22 \mathrm{~A}$ & 121.24 EFC & $116.12 \mathrm{KL}$ & $110.33 \mathrm{~N}$ & $106.43 \mathrm{P}$ & $115.87 \mathrm{E}$ \\
\hline \multirow[t]{4}{*}{ Polyethylene } & B & $125.22 \mathrm{~A}$ & 123.24 CD & $120.43 \mathrm{FG}$ & $117.04 \mathrm{~K}$ & $115.30 \mathrm{LM}$ & $120.25 \mathrm{C}$ \\
\hline & C & $125.22 \mathrm{~A}$ & $123.90 \mathrm{~B}-\mathrm{D}$ & $122.15 \mathrm{E}$ & $119.43 \mathrm{HI}$ & $116.65 \mathrm{~K}$ & 121.47 B \\
\hline & Mean & $125.22 \mathrm{~A}$ & $122.79 \mathrm{~B}$ & 119.57 D & $115.60 \mathrm{~F}$ & $112.79 \mathrm{G}$ & $119.19 \mathrm{~B}$ \\
\hline & A & $125.22 \mathrm{~A}$ & $121.24 \mathrm{EF}$ & $118.75 \mathrm{IJ}$ & $114.65 \mathrm{M}$ & 109.030 & $117.78 \mathrm{D}$ \\
\hline \multirow[t]{4}{*}{ Polypropylene } & B & $125.22 \mathrm{~A}$ & $124.14 \mathrm{BC}$ & $122.15 \mathrm{E}$ & $119.72 \mathrm{GH}$ & $118.27 \mathrm{~J}$ & $121.90 \mathrm{~B}$ \\
\hline & C & $125.22 \mathrm{~A}$ & $125.22 \mathrm{AB}$ & $123.14 \mathrm{D}$ & $121.74 \mathrm{E}$ & $120.46 \mathrm{FG}$ & 123.16 A \\
\hline & Mean & 125.22 A & $123.53 \mathrm{~B}$ & $121.35 \mathrm{C}$ & 118.70 E & $115.92 \mathrm{~F}$ & $120.94 \mathrm{~A}$ \\
\hline & A & $125.22 \mathrm{~A}$ & 121.24 DE & $117.44 \mathrm{G}$ & $112.49 \mathrm{H}$ & 107.73 I & $116.82 \mathrm{C}$ \\
\hline Mean & B & $125.22 \mathrm{~A}$ & $123.69 \mathrm{~B}$ & $121.29 \mathrm{D}$ & $118.38 \mathrm{~F}$ & $116.79 \mathrm{G}$ & $121.07 \mathrm{~B}$ \\
\hline \multirow[t]{4}{*}{ of ( MAP) } & C & $125.22 \mathrm{~A}$ & $124.56 \mathrm{~B}$ & $122.65 \mathrm{C}$ & $120.59 \mathrm{E}$ & $118.56 \mathrm{~F}$ & 122.31 A \\
\hline & Mean & $125.22 \mathrm{~A}$ & 123.16 B & $120.46 \mathrm{C}$ & 117.15 D & $114.36 \mathrm{E}$ & \\
\hline & & \multicolumn{5}{|c|}{2010 season } & \\
\hline & A & $123.24 \mathrm{~A}$ & 118.73 EF & $113.79 \mathrm{KL}$ & $108.11 \mathrm{~N}$ & 104.30 P & $113.63 \mathrm{E}$ \\
\hline \multirow[t]{4}{*}{ Polyethylene } & B & $123.24 \mathrm{~A}$ & $120.75 \mathrm{C}$ & $118.00 \mathrm{FG}$ & $114.68 \mathrm{~K}$ & $112.98 \mathrm{LM}$ & $117.93 \mathrm{C}$ \\
\hline & C & $123.24 \mathrm{~A}$ & 121.42 BC & 119.70 D & $117.02 \mathrm{HI}$ & $114.33 \mathrm{~K}$ & 119.14 B \\
\hline & Mean & 123.24 A & $120.30 \mathrm{~B}$ & 117.16 D & 113.27 F & $110.54 \mathrm{G}$ & 116.90 B \\
\hline & A & $123.24 \mathrm{~A}$ & 118.82 DEF & $116.35 \mathrm{IJ}$ & $112.33 \mathrm{M}$ & 106.830 & 115.51 D \\
\hline \multirow[t]{4}{*}{ Polypropylene } & B & $123.24 \mathrm{~A}$ & $121.60 \mathrm{BC}$ & $119.70 \mathrm{D}$ & $117.33 \mathrm{GH}$ & $115.90 \mathrm{~J}$ & $119.55 \mathrm{~B}$ \\
\hline & C & $123.24 \mathrm{~A}$ & $121.73 \mathrm{~B}$ & $120.66 \mathrm{C}$ & 119.31 DE & 118.05 FG & $120.60 \mathrm{~A}$ \\
\hline & Mean & 123.24 A & 120.72 B & $118.90 \mathrm{C}$ & $.32 \mathrm{E}$ & $113.59 \mathrm{~F}$ & 118.56 A \\
\hline & A & $123.24 \mathrm{~A}$ & 118.78 DE & $115.07 \mathrm{G}$ & $110.22 \mathrm{H}$ & 105.57 I & $114.57 \mathrm{C}$ \\
\hline Mean & B & $123.24 \mathrm{~A}$ & $121.18 \mathrm{~B}$ & 118.85 D & $116.01 \mathrm{~F}$ & $114.44 \mathrm{G}$ & 118.74 B \\
\hline \multirow[t]{2}{*}{ of ( MAP) } & C & $123.24 \mathrm{~A}$ & $121.58 \mathrm{~B}$ & $120.18 \mathrm{C}$ & 118.17 E & $116.19 \mathrm{~F}$ & 119.87 A \\
\hline & Mean & 123.24 A & $120.51 \mathrm{~B}$ & $118.03 \mathrm{C}$ & 114.80 D & $112.07 \mathrm{E}$ & \\
\hline
\end{tabular}

Means in the same column having the same letter are not significantly different at 0.05 level by Duncan's multiple rang test.

$A=P a s s i v e$ MAP

$\mathrm{B}=$ Active $\mathrm{MAP}(5 \% 02+5 \% \mathrm{Co} 2)$

C=Active MAP(5\%02+10\%Co2)

\section{Total chlorophyll:}

Data in Table (6) showed that chlorophyll content in broccoli florets decreased gradually during storage. This decrement could be attributed to the gradual increase of destruction by chlorophyll degrading peroxide (POD) activity which is t transformation of chloroplasts to chromplasts (Charles et al., 1991). 
Concerning the effect of packaging materials, data revealed that the highest content of chlorophyll was obtained from broccoli florets packed in polypropylene film.

Table(6): Effect of active and passive modified atmosphere packages (MAP) on total chlorophyll(mg/100mg f.w) of broccoli florets during storage in 2009 and 2010 seasons.

\begin{tabular}{|c|c|c|c|c|c|c|c|}
\hline \multirow{2}{*}{\begin{tabular}{|c|} 
Packaging \\
materials \\
\end{tabular}} & \multirow{2}{*}{ MAP } & \multicolumn{5}{|c|}{ storage period (days) } & \multirow[t]{2}{*}{ Mean } \\
\hline & & 0 & 4 & 8 & 12 & 16 & \\
\hline & & \multicolumn{5}{|c|}{2009 season } & \\
\hline & A & $103.25 \mathrm{~A}$ & 100.08 D & $96.75 \mathrm{G}$ & $93.29 \mathrm{~J}$ & $89.24 \mathrm{M}$ & $96.52 \mathrm{~F}$ \\
\hline Polyethyle & B & $103.25 \mathrm{~A}$ & $101.23 \mathrm{C}$ & $99.27 \mathrm{E}$ & $95.41 \mathrm{H}$ & $91.27 \mathrm{~K}$ & $98.09 \mathrm{D}$ \\
\hline & C & $103.25 \mathrm{~A}$ & $101.98 \mathrm{~B}$ & $101.10 \mathrm{C}$ & $97.43 \mathrm{~F}$ & 94.351 & $99.62 \mathrm{~B}$ \\
\hline & Mean & $103.25 \mathrm{~A}$ & $101.10 \mathrm{C}$ & $99.04 \mathrm{E}$ & $95.38 \mathrm{G}$ & 91.63 I & $98.08 \mathrm{~B}$ \\
\hline & A & $103.25 \mathrm{~A}$ & 100.24 D & $97.42 \mathrm{~F}$ & $95.19 \mathrm{H}$ & $90.37 \mathrm{~L}$ & $97.29 \mathrm{E}$ \\
\hline \multicolumn{2}{|c|}{ Polypropylene B } & $103.25 \mathrm{~A}$ & $102.27 \mathrm{~B}$ & $100.22 \mathrm{D}$ & $97.27 \mathrm{~F}$ & $93.31 \mathrm{~J}$ & $99.26 \mathrm{C}$ \\
\hline & C & $103.25 \mathrm{~A}$ & $102.14 \mathrm{~B}$ & $101.26 \mathrm{C}$ & $99.4 \mathrm{E}$ & $97.32 \mathrm{~F}$ & $100.68 \mathrm{~A}$ \\
\hline & Mean & $103.25 \mathrm{~A}$ & $101.55 \mathrm{~B}$ & $99.63 \mathrm{D}$ & 97.39 F & $93.67 \mathrm{H}$ & $99.08 \mathrm{~A}$ \\
\hline & A & $103.25 \mathrm{~A}$ & 100.16 E & $97.09 \mathrm{H}$ & $94.24 \mathrm{~K}$ & $89.81 \mathrm{M}$ & $96.91 \mathrm{C}$ \\
\hline Mean & B & $103.25 \mathrm{~A}$ & $101.75 \mathrm{C}$ & $99.75 \mathrm{~F}$ & 96.341 & $92.29 \mathrm{~L}$ & $98.68 \mathrm{~B}$ \\
\hline \multirow[t]{4}{*}{ of ( MAP) } & C & $103.25 \mathrm{~A}$ & $102.60 \mathrm{~B}$ & $101.18 \mathrm{D}$ & $98.42 \mathrm{G}$ & $95.84 \mathrm{~J}$ & $100.15 \mathrm{~A}$ \\
\hline & Mean & 103.25 A & 101.32 B & $99.34 \mathrm{C}$ & 96.33 D & $92.64 \mathrm{E}$ & \\
\hline & & \multicolumn{5}{|c|}{2010 season } & \\
\hline & A & $101.82 \mathrm{~A}$ & $98.05 \mathrm{FG}$ & $95.30 \mathrm{~J}$ & $92.39 \mathrm{M}$ & 89.240 & $95.36 \mathrm{E}$ \\
\hline \multirow[t]{4}{*}{ Polyethylen } & B & $101.82 \mathrm{~A}$ & $99.98 \mathrm{D}$ & $97.78 \mathrm{G}$ & $94.55 \mathrm{~K}$ & $91.28 \mathrm{~N}$ & $97.08 \mathrm{C}$ \\
\hline & C & $101.82 \mathrm{~A}$ & $100.73 \mathrm{C}$ & $99.59 \mathrm{DE}$ & 96.46 I & $93.43 \mathrm{~L}$ & $98.41 \mathrm{~B}$ \\
\hline & Mean & $101.82 \mathrm{~A}$ & $99.59 \mathrm{C}$ & $97.56 \mathrm{E}$ & $94.47 \mathrm{G}$ & 91.32 I & $96.95 \mathrm{~B}$ \\
\hline & A & $101.82 \mathrm{~A}$ & $99.31 \mathrm{E}$ & $96.93 \mathrm{H}$ & $93.05 \mathrm{~L}$ & 89.370 & 96.10 D \\
\hline \multicolumn{2}{|c|}{ Polypropylene B } & $101.82 \mathrm{~A}$ & $101.33 \mathrm{~B}$ & $99.72 \mathrm{D}$ & 96.12 I & $93.31 \mathrm{~L}$ & 98.46 B \\
\hline & C & $101.82 \mathrm{~A}$ & $101.53 \mathrm{AB}$ & $100.73 \mathrm{C}$ & $98.28 \mathrm{~F}$ & 96.371 & $99.75 \mathrm{~A}$ \\
\hline & Mean & $101.82 \mathrm{~A}$ & 100.72 B & 99.13 D & $95.82 \mathrm{~F}$ & $93.02 \mathrm{H}$ & $98.10 \mathrm{~A}$ \\
\hline & A & $101.82 \mathrm{~A}$ & $98.68 \mathrm{E}$ & $96.12 \mathrm{G}$ & $92.72 \mathrm{~J}$ & $89.31 \mathrm{~L}$ & $95.73 \mathrm{C}$ \\
\hline Mean & B & $101.82 \mathrm{~A}$ & $100.66 \mathrm{C}$ & $98.75 \mathrm{E}$ & $95.34 \mathrm{H}$ & $92.30 \mathrm{~K}$ & 97.77 B \\
\hline \multirow[t]{2}{*}{ of (MAP) } & C & $101.82 \mathrm{~A}$ & 101.13 B & 100.16 D & $97.37 \mathrm{~F}$ & 94.901 & $99.08 \mathrm{~A}$ \\
\hline & Mean & $101.82 \mathrm{~A}$ & 100.16 B & $98.34 \mathrm{C}$ & 95.14 D & $92.17 \mathrm{E}$ & \\
\hline
\end{tabular}

Means in the same column having the same letter are not significantly different at 0.05 level by Duncan's multiple rang test.

A=Passive MAP

B=Active MAP(5\%02+5\%Co2)

C=Active MAP(5\%02+10\%Co2)

Florets packed in active MAP retained more chlorophyll content compared with those of passive MAP which gave the lowest ones. The most important delay in chlorophyll degradation was observed in florets exposed to $5 \% \mathrm{O}_{2}+10 \mathrm{Co}_{2}$.

The interaction of packaging material, MAP and storage period was significant after 16 days of storage. The lowest value of chlorophyll contents was noted in broccoli florets packed in polyethylene film plus passive MAP, while the highest one was found in broccoli packed in polypropylene film plus active MAP at $5 \% \mathrm{O}_{2}+10 \% \mathrm{Co}_{2}$ during the same period. 
Gas composition inside the packages:

Since broccoli is still alive after harvest it also respires. It is necessary to achieve proper gas composition in the packages, so it is very important to study the gas changes inside the package of MAP.

Moreover, the atmosphere analysis showed that, in two packages, the atmosphere had been modified (Tables 7\&8). The $\mathrm{O}_{2}$ and $\mathrm{Co}_{2}$ inside the packages differed significantly between packaging materials. Broccoli florets packed in polyethylene film had low $\mathrm{O}_{2}$ and high $\mathrm{Co}_{2}$ as comported with those in polypropylene ones. The $\mathrm{O}_{2}$ concentration was above the critical concentration (0.5-2.5\%) for developing off-odor (Ballantyne et al., 1988; Makhlouf et al., 1989).

Table(7): Effect of active and passive modified atmosphere packages (MAP) on $\mathrm{O}_{2}$ concentration inside the packages of broccoli florets during storage in 2009 and 2010 seasons.

\begin{tabular}{|c|c|c|c|c|c|c|c|}
\hline \multirow{4}{*}{$\begin{array}{l}\text { Packaging } \\
\text { materials }\end{array}$} & \multirow{2}{*}{ MAP } & \multicolumn{5}{|c|}{ storage period (days) } & \multirow{2}{*}{ Mean } \\
\hline & & 0 & 4 & 8 & 12 & 16 & \\
\hline & & \multicolumn{5}{|c|}{2009 season } & \\
\hline & A & $20.80 \mathrm{~A}$ & $19.00 \mathrm{C}$ & $15.45 \mathrm{E}$ & $12.37 \mathrm{~F}$ & $10.00 \mathrm{G}$ & $15.52 \mathrm{~B}$ \\
\hline \multirow[t]{4}{*}{ Polyethylene } & B & $5.00 \mathrm{H}$ & $3.60 \mathrm{~L}$ & $3.00 \mathrm{M}$ & $2.80 \mathrm{M}$ & $2.20 \mathrm{~N}$ & $3.32 \mathrm{E}$ \\
\hline & C & $5.00 \mathrm{H}$ & $4.60 \mathrm{HI}$ & $4.40 \mathrm{lJ}$ & $4.00 \mathrm{~J}-\mathrm{L}$ & $2.00 \mathrm{~N}$ & $4.00 \mathrm{D}$ \\
\hline & Mean & 10.27 A & 9.07 D & $7.62 \mathrm{~F}$ & $6.39 \mathrm{G}$ & $4.73 \mathrm{H}$ & $7.61 \mathrm{~B}$ \\
\hline & A & $20.80 \mathrm{~A}$ & $20.37 \mathrm{~A}$ & $19.60 \mathrm{~B}$ & $17.87 \mathrm{D}$ & $15.07 \mathrm{E}$ & 18.74 A \\
\hline \multirow[t]{4}{*}{ Polypropylene } & B & $5.00 \mathrm{H}$ & $4.63 \mathrm{HI}$ & $4.43 \mathrm{IJ}$ & $4.00 \mathrm{~J}-\mathrm{L}$ & $3.70 \mathrm{KL}$ & $4.35 \mathrm{C}$ \\
\hline & C & $5.00 \mathrm{H}$ & $4.70 \mathrm{HI}$ & $4.60 \mathrm{HI}$ & $4.13 \mathrm{JK}$ & $3.93 \mathrm{KL}$ & $4.47 \mathrm{C}$ \\
\hline & Mean & $10.27 \mathrm{~A}$ & $9.90 \mathrm{~B}$ & $9.54 \mathrm{C}$ & $8.67 \mathrm{E}$ & $7.57 \mathrm{~F}$ & $9.19 \mathrm{~A}$ \\
\hline & A & $20.80 \mathrm{~A}$ & $19.69 \mathrm{~B}$ & $17.53 \mathrm{C}$ & 15.12 D & $12.54 \mathrm{E}$ & $17.13 \mathrm{~A}$ \\
\hline Mean & B & $5.00 \mathrm{~F}$ & $4.12 \mathrm{H}$ & 3.721 & 3.401 & $2.95 \mathrm{~J}$ & $3.84 \mathrm{C}$ \\
\hline \multirow[t]{4}{*}{ of ( MAP) } & C & $5.00 \mathrm{~F}$ & $4.65 \mathrm{G}$ & $4.50 \mathrm{G}$ & $4.07 \mathrm{H}$ & $2.97 \mathrm{~J}$ & $4.24 \mathrm{~B}$ \\
\hline & Mean & $10.27 \mathrm{~A}$ & $9.48 \mathrm{~B}$ & $8.58 \mathrm{C}$ & $7.53 \mathrm{D}$ & $6.15 \mathrm{E}$ & \\
\hline & & \multicolumn{5}{|c|}{2010 season } & \\
\hline & A & $20.80 \mathrm{~A}$ & $18.83 \mathrm{D}$ & $14.50 \mathrm{~F}$ & $11.30 \mathrm{G}$ & $10.20 \mathrm{H}$ & 15.13 B \\
\hline \multirow[t]{4}{*}{ Polyethylene } & B & 5.001 & $4.30 \mathrm{KL}$ & 4.13 L-N & 3.570 & $2.10 \mathrm{P}$ & $3.82 \mathrm{D}$ \\
\hline & C & 5.001 & $4.47 \mathrm{JKL}$ & $4.30 \mathrm{KL}$ & 3.700 & $1.87 \mathrm{P}$ & 3.87 D \\
\hline & Mean & $10.27 \mathrm{~A}$ & $9.20 \mathrm{C}$ & $7.64 \mathrm{E}$ & $6.19 \mathrm{G}$ & $4.72 \mathrm{H}$ & $7.60 \mathrm{~B}$ \\
\hline & A & $20.80 \mathrm{~A}$ & $20.20 \mathrm{~B}$ & $19.30 \mathrm{C}$ & $17.17 \mathrm{E}$ & $14.70 \mathrm{~F}$ & $18.43 \mathrm{~A}$ \\
\hline \multirow[t]{4}{*}{ Polypropylene } & B & $5.00 \mathrm{I}$ & $4.63 \mathrm{I}-\mathrm{K}$ & 4.23 LM & $3.77 \mathrm{N0}$ & 3.500 & $4.23 \mathrm{C}$ \\
\hline & C & 5.001 & $4.70 \mathrm{IJ}$ & $4.37 \mathrm{~J}-\mathrm{L}$ & $3.87 \mathrm{M}-0$ & 3.730 & $4.33 \mathrm{C}$ \\
\hline & Mean & 10.27 A & $9.84 \mathrm{~B}$ & $9.30 \mathrm{C}$ & 8.27 D & $7.31 \mathrm{~F}$ & $9.00 \mathrm{~A}$ \\
\hline & A & $20.80 \mathrm{~A}$ & $19.52 \mathrm{~B}$ & $16.90 \mathrm{C}$ & $14.24 \mathrm{D}$ & $12.45 \mathrm{E}$ & $16.78 \mathrm{~A}$ \\
\hline \multicolumn{2}{|l|}{ Mean } & $5.00 \mathrm{~F}$ & $4.47 \mathrm{G}$ & $4.18 \mathrm{H}$ & 3.671 & $2.80 \mathrm{~J}$ & 4.02 B \\
\hline \multirow[t]{2}{*}{ of ( MAP) } & C & $5.00 \mathrm{~F}$ & $4.59 \mathrm{G}$ & $4.34 \mathrm{GH}$ & 3.791 & $2.80 \mathrm{~J}$ & $4.10 \mathrm{~B}$ \\
\hline & Mean & 10.27 A & 9.52 B & $8.47 \mathrm{C}$ & 7.23 D & $6.02 \mathrm{E}$ & \\
\hline
\end{tabular}

Means in the same column having the same letter are not significantly different at 0.05 level by Duncan's multiple rang test.

A=Passive MAP

B=Active MAP $(5 \% 02+5 \%$ Co2 $)$

C=Active MAP(5\%02+10\%Co2) 
Table(8): Effect of active and passive modified atmosphere packages (MAP) on $\mathrm{CO}_{2}$ concentration inside the packages of broccoli florets during storage in 2009 and 2010 seasons.

\begin{tabular}{|c|c|c|c|c|c|c|c|}
\hline \multirow{4}{*}{$\begin{array}{c}\text { Packaging } \\
\text { materials }\end{array}$} & \multirow{2}{*}{ MAP } & \multicolumn{5}{|c|}{ storage period (days) } & \multirow{2}{*}{ Mean } \\
\hline & & 0 & 4 & 8 & 12 & 16 & \\
\hline & & \multicolumn{5}{|c|}{2009 season } & \\
\hline & A & $0.03 \mathrm{~T}$ & $2.20 \mathrm{R}$ & $3.57 \mathrm{Q}$ & $5.73 \mathrm{M}$ & $7.20 \mathrm{~K}$ & $3.75 \mathrm{E}$ \\
\hline \multirow[t]{4}{*}{ Polyethylene } & B & $5.00 \mathrm{~N}$ & $6.00 \mathrm{M}$ & $10.00 \mathrm{I}$ & $12.07 \mathrm{E}$ & $15.27 \mathrm{C}$ & $9.67 \mathrm{C}$ \\
\hline & C & $10.00 I$ & $11.40 \mathrm{~F}$ & $13.07 \mathrm{D}$ & $16.00 \mathrm{~B}$ & $19.77 \mathrm{~A}$ & $14.05 \mathrm{~A}$ \\
\hline & Mean & $5.01 \mathrm{H}$ & $6.53 \mathrm{~F}$ & $8.88 \mathrm{C}$ & 11.27 B & $14.08 \mathrm{~A}$ & $9.15 \mathrm{~A}$ \\
\hline & A & $0.03 \mathrm{~T}$ & $1.30 \mathrm{~S}$ & $2.43 \mathrm{R}$ & $3.93 \mathrm{P}$ & 4.500 & $2.44 \mathrm{~F}$ \\
\hline \multirow[t]{4}{*}{ Polypropylene } & B & $5.00 \mathrm{~N}$ & $5.70 \mathrm{M}$ & $6.67 \mathrm{~L}$ & $7.30 \mathrm{~K}$ & $8.53 \mathrm{~J}$ & $6.64 \mathrm{D}$ \\
\hline & C & $10.00 \mathrm{I}$ & $10.40 \mathrm{H}$ & $10.90 \mathrm{G}$ & $11.37 \mathrm{~F}$ & $11.93 \mathrm{E}$ & 10.92 B \\
\hline & Mean & $5.01 \mathrm{H}$ & $5.80 \mathrm{G}$ & $6.67 \mathrm{~F}$ & $7.53 \mathrm{E}$ & 8.32 D & $6.67 \mathrm{~B}$ \\
\hline & A & $0.03 \mathrm{~L}$ & $1.75 \mathrm{~K}$ & $3.00 \mathrm{~J}$ & 4.831 & $5.85 \mathrm{H}$ & $3.09 \mathrm{C}$ \\
\hline Mean & B & 5.001 & $5.85 \mathrm{H}$ & $8.34 \mathrm{G}$ & $9.69 \mathrm{~F}$ & $11.90 \mathrm{C}$ & $8.15 \mathrm{~B}$ \\
\hline \multirow[t]{4}{*}{ of ( MAP) } & C & $10.00 \mathrm{E}$ & $10.90 \mathrm{D}$ & $11.99 \mathrm{C}$ & $13.69 \mathrm{~B}$ & $15.85 \mathrm{~A}$ & $12.48 \mathrm{~A}$ \\
\hline & Mean & $5.01 \mathrm{E}$ & 6.17 D & $7.77 \mathrm{C}$ & $9.40 \mathrm{~B}$ & $11.20 \mathrm{~A}$ & \\
\hline & \multicolumn{6}{|c|}{2010 season } & \\
\hline & A & $0.03 \mathrm{~N}$ & 2.30 LM & $4.07 \mathrm{~K}$ & 5.801 & $7.30 \mathrm{H}$ & $3.90 \mathrm{E}$ \\
\hline \multirow[t]{4}{*}{ Polyethylene } & B & $5.00 \mathrm{I}-\mathrm{K}$ & $7.07 \mathrm{H}$ & 10.07 FG & $13.17 \mathrm{D}$ & $15.47 \mathrm{C}$ & 10.16 C \\
\hline & C & $10.00 \mathrm{FG}$ & $11.50 \mathrm{E}$ & 14.17 D & $17.17 \mathrm{~B}$ & $19.07 \mathrm{~A}$ & $14.38 \mathrm{~A}$ \\
\hline & Mean & $5.01 \mathrm{H}$ & 6.96 EF & $9.44 \mathrm{C}$ & $12.05 \mathrm{~B}$ & $13.95 \mathrm{~A}$ & $9.48 \mathrm{~A}$ \\
\hline & A & $0.03 \mathrm{~N}$ & $1.30 \mathrm{M}$ & $2.73 \mathrm{~L}$ & $3.97 \mathrm{~K}$ & $4.63 \mathrm{JK}$ & $2.53 \mathrm{~F}$ \\
\hline \multirow[t]{4}{*}{ Polypropylene } & B & $5.00 \mathrm{I}-\mathrm{K}$ & $5.43 \mathrm{IJ}$ & 5.901 & $7.40 \mathrm{H}$ & $9.83 \mathrm{G}$ & 6.71 D \\
\hline & C & 10.00 FG & 10.53 E-G & 10.70 E-G & 11.13 EF & $11.57 \mathrm{E}$ & 10.79 B \\
\hline & Mean & $5.01 \mathrm{H}$ & $5.75 \mathrm{G}$ & $6.44 \mathrm{~F}$ & $7.50 \mathrm{E}$ & 8.68 D & $6.68 \mathrm{~B}$ \\
\hline & A & $0.03 \mathrm{~K}$ & $1.80 \mathrm{~J}$ & 3.401 & $4.89 \mathrm{H}$ & $5.97 \mathrm{G}$ & $3.22 \mathrm{C}$ \\
\hline Mean & B & $5.00 \mathrm{H}$ & $6.25 \mathrm{G}$ & $7.99 \mathrm{~F}$ & 10.29 DE & $12.65 \mathrm{C}$ & $8.432 \mathrm{~B}$ \\
\hline \multirow[t]{2}{*}{ of ( MAP) } & C & $10.00 \mathrm{E}$ & $11.02 \mathrm{D}$ & $12.44 \mathrm{C}$ & $14.15 \mathrm{~B}$ & $15.32 \mathrm{~A}$ & $12.58 \mathrm{~A}$ \\
\hline & Mean & $5.01 \mathrm{E}$ & 6.36 D & $7.94 \mathrm{C}$ & 9.77 B & $11.31 \mathrm{~A}$ & \\
\hline
\end{tabular}

Means in the same column having the same letter are not significantly different at 0.05 level by Duncan's multiple rang test.

A=Passive MAP

B=Active MAP $(5 \% 02+5 \%$ Co2)

C=Active MAP(5\%02+10\%Co2)

The $\mathrm{O}_{2}$ levels in active MAP were lower than that of passive MAP as shown in Table (7). While the values of $\mathrm{Co}_{2}$ were higher (Table 8). The $\mathrm{O}_{2}$ level decreased and $\mathrm{Co}_{2}$ increased continuously until reached $6.09 \% \mathrm{O}_{2}$ and $11.26 \% \mathrm{Co}_{2}$ after 16 days of storage at $0^{\circ} \mathrm{C}$ (average of the two seasons).

After 16 days at $0^{\circ} \mathrm{C}$, the gas concentrations were $2.88 \% \mathrm{O}_{2} \& 12.28 \%$ $\mathrm{Co}_{2}$ and $2.89 \% \mathrm{O}_{2} \& 15.59 \% \mathrm{Co}_{2}$ (average of the two seasons) in active MAP at $5 \% \mathrm{O}_{2}+5 \% \mathrm{Co}_{2}$ and $5 \% \mathrm{O}_{2}+10 \% \mathrm{CO}_{2}$ respectively. The gas composition inside the passive package reached $12.5 \% \mathrm{O}_{2} \& 5.91 \% \mathrm{Co}_{2}$ in the same period. 


\section{CONCLUSION}

Broccoli florets packed in polypropylene film and exposed to active MAP $\left(5 \% \mathrm{O}_{2}+10 \% \mathrm{Co}_{2}\right)$ were the most effective treatment for reducing weight loss and color change and maintaining high content of chlorophyll and gave florets with a good appearance for 16 days at $0^{\circ} \mathrm{C}$ and $95 \% \mathrm{RH}$.

\section{REFERENCES}

A.O.A.C. 1990. Official Methods of Analysis Association of Official Analytical Chemists 13 the ed. Washington, S.C., U.S.A.

Ballantyne, A., R. Stark and J. D. Selman .1988. Modified atmosphere packaging of broccoli florets. International Journal of Food Science and Technology. 23:353-360

Barth, M. M., F. L. Kerbel, A. K. Perry and S. J. Schmidt .1993. Modified atmosphere packaging effect ascorbic acid, enzyme activity and market quality of broccoli. Journal of Food Science. 58(1): 140-143.

Brennan, P. S. and R. L. Shewfelt .1989. Effect of cooling delay at harvest on broccoli quality during postharvest storage.J.Food Qual. 12:13-22.

Charles, F., C. F. Forney and R. E. Rij .1991. Temperature of broccoli florets at time of packaging influences package. HortScience.26:1301-1303.

Dong, H., Y. Jiang, Y. Wang, R. Liu and H. Guan.2004. Effects of Hot Water Immersion on Storage Quality of Fresh Broccoli Heads. Food T echnol. Biotechnol. 42(2):135-139.

Elkashif, M. E., D. J. Huber and M. Sherman .1993. Delaying deterioration of broccoli and cucumber using polymeric films.Proc. Fla. Syate Hort. Soc. 96:332-335.

Forney, C.F. and R. E. Rij.1991. Temperature of broccoli florets at time of packaging influences package. HortScience.26:1301-1303.

Hansen, M., R. G. Buttery, D. J. Stern, M. I. Cantwell, and L.C. Ling .1992. Broccoli storage under low-oxygen atmosphere identification of higher boiling volatiles. Journal of Agricultural Food Chemistry. 40(5):850-852.

Hansen, M., C. E. Olsen, L. Poll, and, M. I. Cantwell .1993. Volatile constituents and sensory quality of cooked broccoli florets after aerobic and anaerobic storage. Postharvest. 92:105-111.

Hu, W.Z., A. L. Jiang, K. Pang and H. P. Qi .2007. Atmosphere composition, respiration rate and quality of fresh-cut cabbages in active modified atmosphere packaging. Proc. IC on Qual. Manag. Fresh Cut Produce. Acta Hort. 746:69-72

Jacobsson, A. and T. Nielsen .2003. Influence of packaging material and storage temperature on the texture, colour, and weight of broccoli. Proc. $8^{\text {th }}$ Int. CA Conference. Acta Hort. 600:323-325.

Jacobsson, A., T. Nielsen and Karin Wendin .2003. Influence of packaging material and storage temperature on the texture, colour, and weight of broccoli. Acta Hort. 599:91-93.

Jacobsson, A., T. Nielsen, I. Sjöholm and K. Wendin .2004. Influence of packaging material and storage condition on the sensory quality of broccoli. Food Quality and Preference. 15:301-310. 
Joseph, H. H. and j. B. Mishael .1992. Influence of new packaging technologies on the growth of microorganisms in produce.Journal of Food protectin.55 (10):815-820

Makhlouf, J., J. A. Castaigne, C. Willemot and A. Gosselin .1989. Long-term storage of broccoli under controlled atmosphere. HortScience. 24(4):637-639.

Ryall, A. L. and W. J. Lipton .1979. Handling, transportation and storage of fruit and vegetables. $2^{\text {nd }}$ ed.AVI, Westport, Conn.

Snedecor, G. W. and W. G. Cochran .1980. Statistical methods.lowa.State Univ. Press. USA.

Wang, C.Y. and H. W. Hruschka .1977. Quality maintenance in polyethelnepackaged broccoli.U.S. Dept. Agr. Mhtg. Res. Rpt.1085.

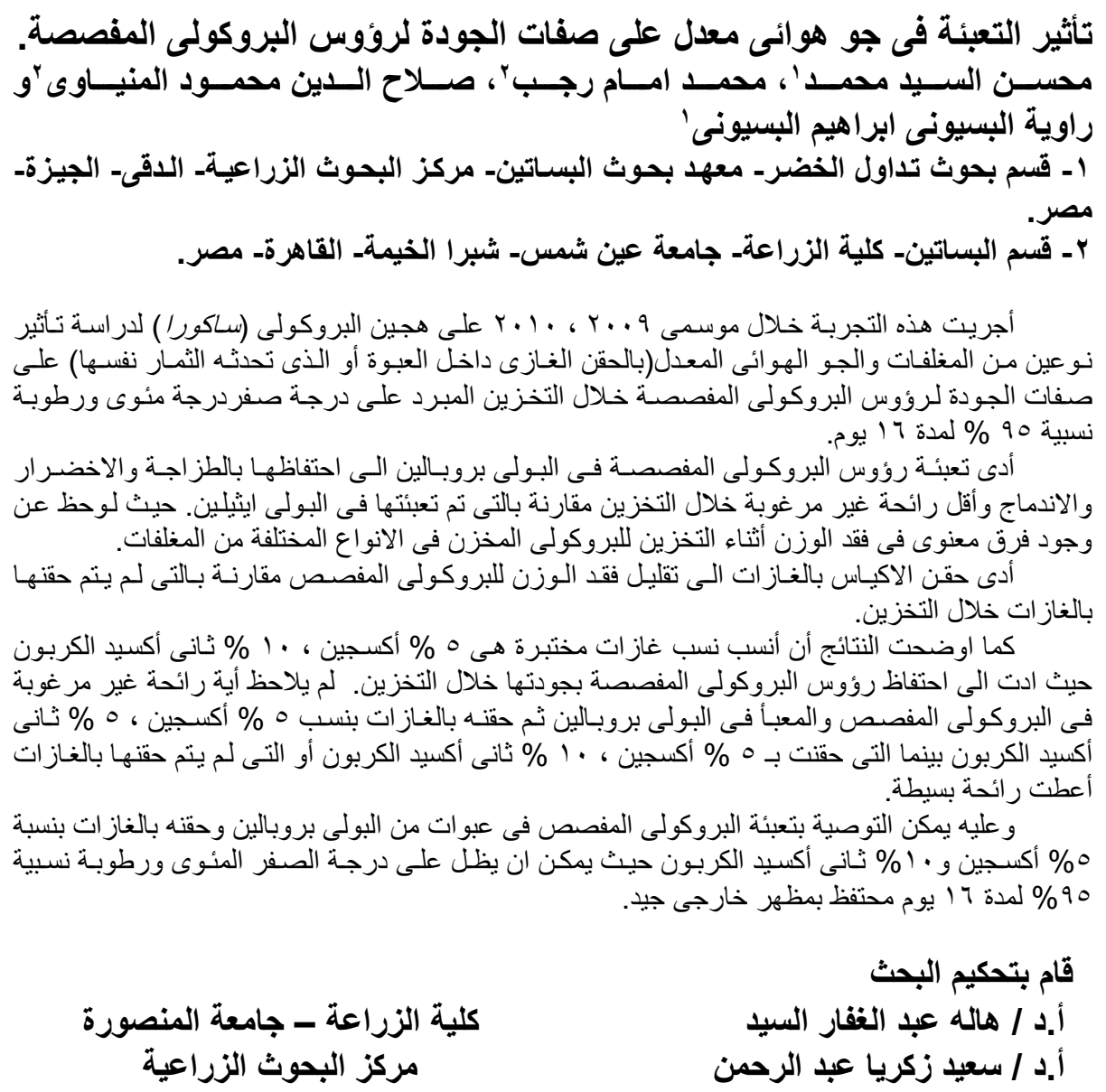

